\title{
EARLY GLOTTIC CARCINOMA: TREATMENT ACCORDING PATIENT'S PREFERENCE?
}

\author{
Sandro J. Stoeckli, MD, ${ }^{1}$ Isabel Schnieper, MD, ${ }^{1}$ Pia Huguenin, MD, ${ }^{2}$ \\ Stephan Schmid, MD ${ }^{1}$
}

${ }^{1}$ Department of Otorhinolaryngology-Head and Neck Surgery, University Hospital Zurich, Frauenklinikstrasse 24, CH-8091 Zurich, Switzerland. E-mail: sandro.stoeckli@usz.ch

${ }^{2}$ Clinic of Radiation Oncology, University Hospital Zurich, Zurich, Switzerland

Accepted 31 March 2003

Published online 23 June 2003 in Wiley InterScience (www.interscience.wiley.com). DOI: 10.1002/hed. 10323

Abstract: Background. To analyze the results in early (stage I and II) glottic carcinoma of a single institution with a patientoriented concept.

Methods. After diagnostic workup and multidisciplinary counseling, either radiotherapy or $\mathrm{CO}_{2}$ laser surgery was chosen with respect to the patient's preference.

Results. Sixty-five patients underwent laser surgery and 75 had radiotherapy. For T1 tumors, the 5-year overall survival, disease-specific survival, and initial local control rate after laser surgery and radiotherapy were comparable (OS, $85 \%$ vs $88 \%$; DSS, $96 \%$ vs $93 \%$; LCR, $86 \%$ vs $85 \%$ ). In contrast, initial local control for T2 tumors was significantly more favorable after surgery (OS $83 \%$ vs $78 \%$; DSS, $83 \%$ vs $88 \%$; LCR, $89 \%$ vs $67 \%$ ). Larynx preservation was significantly higher after surgery in T1 tumors (96\% vs $82 \%$ ) and in T2 tumors (89\% vs $77 \%$ ).

Conclusions. Selection of the treatment modality according to patient's preference results in a comparable control of disease for T1 tumors. The initial local control for T2 tumors is significantly better after surgery. In both stages laser surgery warrants a better larynx preservation rate. ( 2003 Wiley Periodicals, Inc. Head Neck 25: 1051-1056, 2003

Keywords: larynx; carcinoma; radiotherapy; laser; surgery

Correspondence to: J. Stoeckli

๑) 2003 Wiley Periodicals, Inc.
It is still an open debate whether patients with early glottic cancer (stage I and II) should be treated by surgery or radiotherapy. The efficacy with respect to local tumor control has been shown for both modalities in several retrospective evaluations. ${ }^{1-6}$ Most of these results have been achieved in cohorts of patients treated either by surgery or radiation. The lack of comparative prospective data has led to a great variation of management practices. ${ }^{7}$ The therapy decision most often depends on the institution's or surgeon's preference. The policy at the Department of Otorhinolaryngology, Head and Neck Surgery, University Hospital of Zurich, Switzerland, is to mainly base the decision on the patient's preference after multidisciplinary counseling. For early glottic carcinomas, either transoral laser surgery or primary high-dose radiotherapy is offered. Radiotherapy is recommended for patients with tumors not suitable for laser surgery because of anatomic reasons.

The aim of our study was to assess the outcome of each treatment modality group, to compare the results with the literature, and to evalu- 
ate how these results are influenced by the selection process in our multidisciplinary approach.

\section{PATIENTS AND METHODS}

All patients treated at the Department of Otorhinolaryngology, Head and Neck Surgery, University Hospital of Zurich, Switzerland, in curative intent for an early (stage I and II) glottic carcinoma between January, 1, 1990, and December, 31,1998 , were included in a retrospective chart analysis. Patients with a tumor-free follow-up of less than 2 years (five patients) were excluded. Because external partial laryngectomies have been performed only in selected rare cases since the introduction of the $\mathrm{CO}_{2}$ laser and the scope of this analysis was the comparison of laser surgery with radiation, patients undergoing external partial laryngectomies (nine patients) were also excluded. A separate off-study analysis of these cases revealed a comparable outcome to that achieved in the laser group (data not shown). As study endpoints, overall survival, disease-specific survival, initial local control rate, and larynx preservation rate at 2 and 5 years were evaluated.

The initial staging included panendoscopy with the patient under general anesthesia and a neck CT scan in most patients. Primary treatment options were either transoral microendoscopic laser surgery or percutaneous radiotherapy. The patients were not randomly assigned to one of the treatment modalities, but both options were offered. Advantages of laser surgery, which were explained to the patients, consisted of the single stage and short duration of the definitive treatment, the possibility of histologic examination of the resection margins, and the preservation of radiotherapy for recurrences or future second primary tumors. According to most published studies ${ }^{8,9}$ assessing objective voice measurements after treatment of early glottic carcinomas, the superiority of the expected voice quality, particularly in larger tumors, was conversely mentioned in favor of the radiotherapy. As to the tumor control rate, both therapy regimens were thought to be equally effective. The final decision for the treatment modality was made together with the patient and his or her relatives after counseling by the head and neck surgeon and the radiation oncologist. Tumors not suitable for laser surgery because of incomplete exposure with the endoscope were treated by radiation. Because this was the case in less than $10 \%$ of the irradiated tumors and separate analysis of these cases did not reveal any differences in the entire group (data not shown), these patients were not excluded from the study.

The surgical procedure was performed with the patient under general anesthesia. The tumor was exposed with a rigid suspension laryngoscope and excised with a microspot $\mathrm{CO}_{2}$ laser under microscopic view. Larger tumors were transected before removal to evaluate the infiltration depth according to the principles published by Steiner. ${ }^{10}$ In case of involvement of the anterior commissure, the tumor was dissected from cranial to caudal in a subperichondrial plane along the inner surface of the thyroid cartilage. If the perichondrium was reached by the tumor, the adjacent piece of thyroid cartilage was also removed. No prophylactic neck dissections were performed, and no patient received postoperative radiotherapy. The follow-up consisted of clinical controls every 6 weeks during the first year, every 3 months during the second year, and every 6 months thereafter. Patients with histologically uncertain resection margins underwent control laryngoscopy under general anesthesia 2 months postoperatively. No regular follow-up CT scans were performed. All patients undergoing radiotherapy had 3-dimensional treatment planning based on a CT in radiotherapy position with the head fixed in a mask. A bolus of $1 \mathrm{~cm}$ wax was used for build-up of the dose. The planning target volume included the larynx and a safety margin according to the extent of the tumor. Before 1995, 70.2 Gy were applied with 1.8 Gy per fraction and six fractions per week (two treatment sessions on 1 day per week; $70 \%$ of the patients), thereafter, the usual total dose was 68 Gy using six sessions of 2.0 Gy per week (30\% of the patients). Both schedules are equally effective.

\section{RESULTS}

The study population consisted of 140 patients (132 men, 8 women), with a mean age of 63 years (range, 41-88 years). No patient was lost to follow-up. Sixty-five patients decided to have their tumor treated surgically, and 75 patients chose radiation therapy. The mean follow-up was 70 months (range, 12-137 months) for the radiotherapy and 60 months (range, 10-125 months) for the surgery group, respectively. The distribution of the initial T stages according to UICC 1997 is shown in Table 1. All tumors were initially staged N0. In the surgical group the anterior commissure was involved in only 3 of 56 patients (5\%) 


\begin{tabular}{lcc}
\hline \multicolumn{3}{c}{ Table 1. Initial T stage } \\
\hline Stage & Radiotherapy $(n=75)$ & $\begin{array}{c}\text { Surgery }(n=65) \\
(\%)\end{array}$ \\
\hline T1 & $45(60)$ & $56(86)$ \\
T2 & $30(40)$ & $9(14)$ \\
\hline
\end{tabular}

with $\mathrm{T} 1$ tumors and in none of the T2 tumor patients. In contrast, in the radiation group 13 of 45 patients $(29 \%)$ with T1 tumors and 10 of 30 patients (33\%) with T2 tumors showed involvement of the anterior commissure.

In the surgery group, seven patients with initial T1 tumors (13\%) and one patient with an initial $\mathrm{T} 2$ tumor (11\%) experienced a local recurrence after a mean interval of 10 months (range 3-36 months). Of these, six patients underwent salvage surgery (three total laryngectomies, two partial laryngectomies, one endoscopic laser cordectomy), and one had salvage radiation therapy. The total laryngectomies had to be performed because of tumor progression to T3 stage. One patient refused any salvage treatment and died 24 months after initial surgery. Despite salvage treatment, three patients died as a result of disease progression (two laryngectomies, one radiotherapy).

Only one patient had a level IV neck recurrence develop 10 months after initial laser treatment and was successfully salvaged by modified radical neck dissection and postoperative radiotherapy.

The 2-year and 5-year overall survival after laser surgery was $96 \%$ and $85 \%$ for $\mathrm{T} 1$ tumors and $100 \%$ and $83 \%$ for T2 tumors. The disease-specific 2 -year and 5-year survival for the surgery group was $96 \%$ and $96 \%$ for T1 tumors and $100 \%$ and $83 \%$ for T2 tumors. The initial local control rates 2 and 5 years after laser surgery were $88 \%$ and $86 \%$ for T1 and $89 \%$ and $89 \%$ for T2 tumors. The Kaplan-Meier estimates are shown in Figures $1-3$. Larynx preservation in the surgery group was achieved in 54 of 56 patients (96\%) with T1 tumors and 8 of 9 patients (89\%) with T2 tumors. In the radiotherapy group, 8 patients with initial $\mathrm{T} 1$ tumors $(18 \%)$ and 10 patients with initial T2 tumors $(33 \%)$ had a local recurrence after a mean time of 11 months (range 3-61 months). Seven of 8 relapsing T1 tumors ( $3 \mathrm{rT} 2,3 \mathrm{rT} 3,1 \mathrm{rT} 4)$ and 4 of 10 relapsing T2 tumors (4 rT4) showed more advanced disease at the point of detection of the recurrence. Therefore, only 2 patients could be salvaged by partial laryngectomy procedures, and

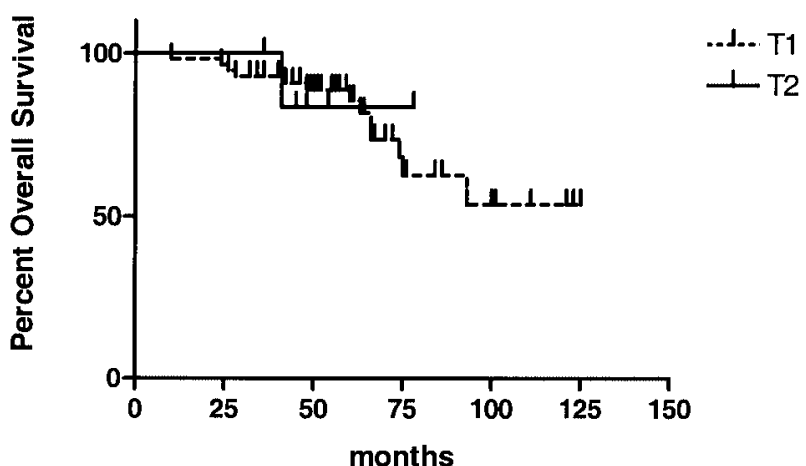

FIGURE 1. Overall survival after endoscopic laser surgery of T1 and T2 glottic carcinomas.

all other 15 patients lost their larynx. Of the 17 salvaged patients, 5 died because of disease progression despite total laryngectomy as salvage treatment.

The 2-year and 5-year overall survival after radiotherapy was $98 \%$ and $88 \%$ for $\mathrm{T} 1$ tumors and $90 \%$ and $78 \%$ for T2 tumors. The disease-specific 2-year and 5-year survival for the radiation group was $98 \%$ and $93 \%$ for T1 tumors and $93 \%$ and $88 \%$ for T2 tumors. The initial local control rates 2 and 5 years after radiotherapy were $85 \%$ and $85 \%$ for T1 and $67 \%$ and $67 \%$ for T2 tumors. the KaplanMeier estimates are shown in Figures 4-6. Larynx preservation in the radiation group was achieved in 37 of $45 \mathrm{~T} 1$ tumor patients (82\%) and in 23 of $30 \mathrm{~T} 2$ tumor patients (77\%). Separate analysis of the radiated patients with anterior commissure involvement versus those without revealed no statistically significant difference in any of the parameters assessed (data not shown).

In $\mathrm{T} 1$ tumors, there was no statistically significant difference (log rank test; $p>.05$ ) for overall survival, disease-specific survival, and local control rate, but a significant difference was

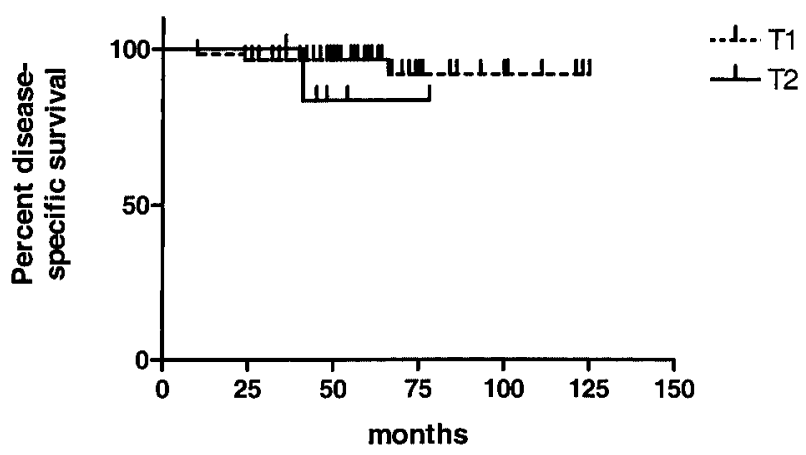

FIGURE 2. Disease-specific survival after endoscopic laser surgery of T1 and T2 glottic carcinomas. 


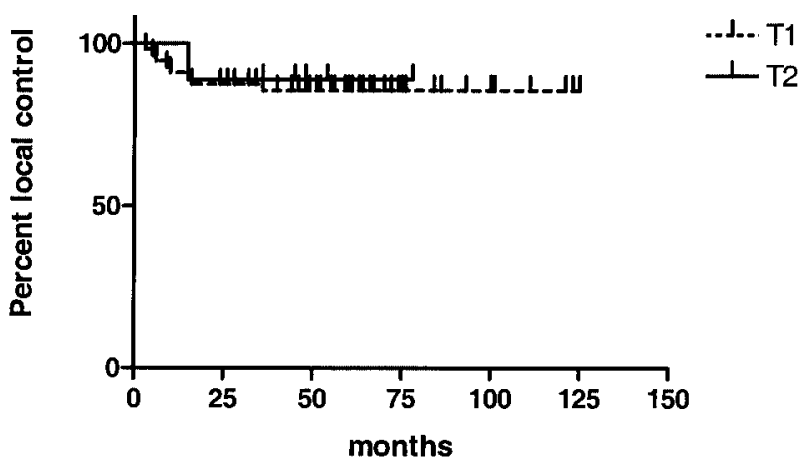

FIGURE 3. Initial local control rate after endoscopic laser surgery of T1 and T2 glottic carcinomas.

found in the larynx preservation rate between radiation and surgery. For T2 tumors, the difference was statistically highly significant (log rank test; $p<.001$ ) for the initial local control rate and the larynx preservation rate but not significant for the other parameters.

\section{DISCUSSION}

The management of early glottic carcinoma is still controversial. DiNardo et $\mathrm{al}^{7}$ investigated the management practices for early laryngeal cancer among members of the American Academy of Otolaryngology-Head and Neck Surgery. More than $50 \%$ of the members completing the questionnaires recommended treating early glottic carcinomas according to the patient's choice. The large variation of practice patterns was explained by the lack of a comparative outcome analysis. It has to be admitted that until recently no prospective randomized trial comparing radiotherapy and laser surgery for early glottic cancer has been published, and the decision in most centers is mainly based on the institution's or surgeon's preference. In Zurich, we offer the patients both options

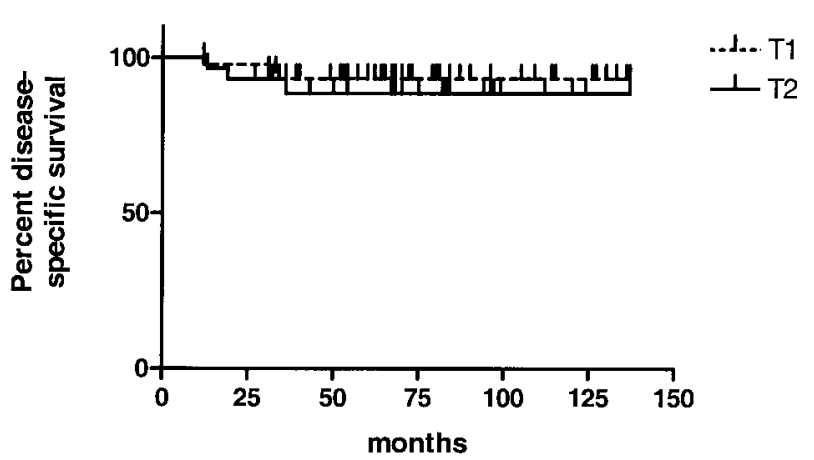

FIGURE 5. Disease-specific survival primary radiotherapy of T1 and T2 glottic carcinomas.

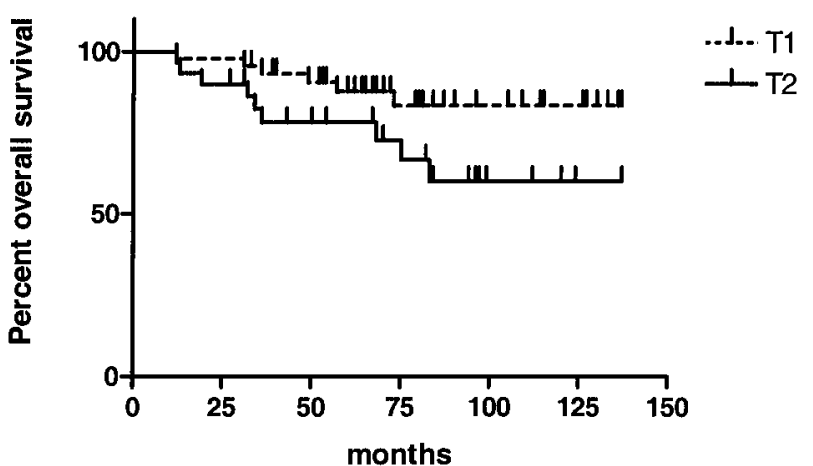

FIGURE 4. Overall survival after primary radiotherapy of $\mathrm{T} 1$ and T2 glottic carcinomas.

and-if oncologically sound-mainly rely on patient's choice. In our opinion, most patients are able and willing to take part in the decisionmaking process. The final decision is the result of a consensus between the patient with his relatives and the head and neck surgeon and radiation oncologist. The decision is made by a wellinformed patient with regard to all patient-, tumor- and treatment-related factors. The challenge of the physician dealing with a cancer patient is to counsel and to help him or her find the most appropriate individual treatment solution. For early glottic carcinoma, there are enough retrospective data in the literature supporting the efficacy of both radiotherapy and laser surgery as the primary treatment. ${ }^{1-6}$ In this situation, not the specific success rate but the differing shortcomings of competing treatment modalities make the difference and have more influence on the patient's decision. Therefore, the aim of our study was to evaluate the results that were achieved with such a multidisciplinary patient-oriented concept at our institution during the last decade.

The two modality groups coming in our cohort

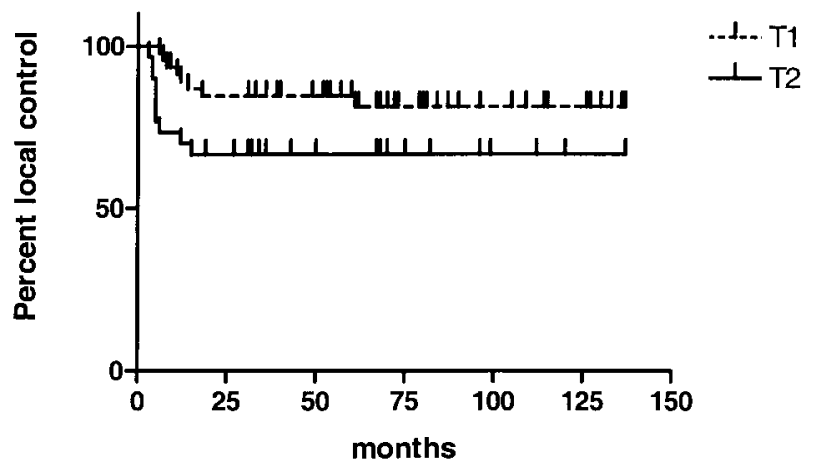

FIGURE 6. Initial local control rate after primary radiotherapy of $\mathrm{T} 1$ and T2 glottic carcinomas. 
of 140 patients were comparable for most factors except the distribution of the $\mathrm{T}$ stages and the involvement of the anterior commissure. Although all efforts were made to objectively counsel the patients, an inadvertent bias for patients with tumors involving the anterior commissure or with larger tumors toward primary radiation seems possible. For this reason, statistical comparison of the two treatment strategies has to be done with caution. The only way to eliminate this problem would be by means of a prospective randomized study.

Involvement of the anterior commissure as a prognostic factor is still debated in the literature. ${ }^{11}$ Recent reports ${ }^{12}$ seem to show that this can not be considered an adverse prognostic factor. In our series, anterior commissure involvement was predominant in the radiation cohort, but within this cohort it was not a prognostic factor.

According to the literature, ${ }^{8,9}$ radiation results in better objective voice results, especially in large tumors. Patients with large tumors and major concerns about voice preferentially chose radiation, resulting in a predominance of $\mathrm{T} 2$ tumors in that group. Patients selecting laser surgery as primary treatment did so because they wanted an expeditious treatment and were not so concerned about their voice. In a recently published study, ${ }^{13}$ we examined the quality of life of patients after treatment of early glottic cancer by means of standardized questionnaires. The patients were also asked to subjectively rate several questions concerning their voice and speech. Surprisingly, there was no statistically significant difference between patients who had undergone laser surgery and those who had received radiation. In conclusion, from the literature, the voice after radiation seems to be better if assessed by sophisticated objective tools, but the patient doesn't feel the same. Excellent 2- and 5-year local control and survival has been achieved for $\mathrm{T} 1$ and $\mathrm{T} 2$ glottic carcinomas with the transoral endoscopic laser. The larynx preservation rate was $95 \%$. Most patients were cured by one single surgical procedure without postoperative radiotherapy. These results are in good agreement with reports by Steiner, ${ }^{4}$ Davis et al, ${ }^{5}$ Perett et al, ${ }^{14}$ and TonVan et $\mathrm{al}^{15}{ }^{15}$ giving 5 -year survival rates for $\mathrm{T} 1$ lesions from 87 to $100 \%$ and for T2 lesions from $78 \%$ to $79 \%$. With regard to our study and the literature, the conclusion can be drawn that endoscopic laser surgery is an excellent treatment option for early glottic carcinomas in patients, who prefer a single-stage, rapid solution.

In the radiation group, equally excellent 2 and 5-year local control was achieved in T1 tumors. However, the larynx preservation rate was lower, indicating that in contrast to laser surgery only few failures after radiation are still accessible for larynx-preserving salvage procedures. ${ }^{16}$ This has to be taken into consideration in the initial counseling of the patients. They have to know that the advantage of good voice quality after radiation bears the risk of larynx loss in case of therapy failure. The initial local control after radiation of T2 tumors was significantly poorer than for T1 tumors and significantly poorer than that achieved with laser surgery (LCR; $89 \%$ vs $67 \%$ ). In $23 \%$ of the patients, a total laryngectomy was necessary to ultimately control the disease. Moose and Greven ${ }^{17}$ in 1997 summarized the radiation results of the most important published studies giving rates of initial local control for T1 lesions from $80 \%$ to $95 \%$ and for T2 lesions from $68 \%$ to $76 \%$. The radiotherapy results of our study are at the bottom line of those published in the literature. Maybe this can be explained by a possible inadvertent selection bias for larger tumors toward radiation, because it is well known from the literature that tumor size is one of the adverse prognostic factors in the radiotherapy of early glottic carcinomas. ${ }^{11,12,18}$ Because this trial was not a prospective randomized one, we cannot answer the question whether the T2 patients as a total would have a better outcome if treated by surgery. But our results suggest that in T2 tumors suitable for laser surgery, this option has to be strongly considered despite its negative implications with voice function.

\section{CONCLUSION}

We can conclude that the management of patients with early glottic carcinoma (stage I and II) as we perform it at our institution seems to select patients suitable for endoscopic laser surgery, giving excellent results for both stages. These patients are cured with a single-stage surgical procedure. Initial tumor control by radiation is comparable only for $\mathrm{T} 1$ tumors but significantly inferior to laser surgery for T2 tumors. In T2 tumors, radiation gives good disease-specific and overall survival, but a considerable number of surgical salvage procedures is needed to secondarily control the disease. Although the patients are counseled as objectively as possible, an inadvertent selection bias for large tumors toward ra- 
diation seems possible and may have contributed to the poorer results of radiation in $\mathrm{T} 2$ tumors. This bias can only be overcome by a prospective randomized trial.

\section{REFERENCES}

1. Mendenhall WM, Parsons JT, Million RR, Fletcher GH. T1-T2 squamous cell carcinoma of the glottic larynx treated with radiation therapy: relationship of dosefractionation factors to local control and complications. Int J Radiat Oncol Biol Phys 1988;15:1267-1273.

2. Mendenhall WM, Million RR, Stringer SP, Cassisi NJ. Squamous cell carcinoma of the glottic larynx: A review emphasizing the University of Florida Philosophy. South Med J 1999;92:385-393.

3. Kelly MD, Hahn SS, Spaulding CA, Kersh CR, Constable WC, Cantrell RW. Definitive radiotherapy in the management of stage I and II carcinomas of the glottis. Ann Otol Rhinol Laryngol 1989;98:235-239.

4. Steiner W. Results of curative laser microsurgery of laryngeal carcinomas. Am J Otolaryngol 1993;14:116-121.

5. Davis RK, Kelly SM, Parkin JL, Stevens MH, Johnson LP. Selective management of early glottic cancer. Laryngoscope 1990;100:1306-1309.

6. McGuirt WF, Koufman JA. Endoscopic laser surgery. Arch Otolaryngol Head Neck Surg 1987;113:501-505.

7. DiNardo LJ, Kaylie DM, Isaacson J. Current treatment practices for early laryngeal carcinoma. Otolaryngol Head Neck Surg 1999;120:30-37.

8. Stewart MG, Chen AY, Stach CB. Outcome analysis of voice and quality of life in patients with laryngeal cancer. Arch Otolaryngol Head Neck Surg 1998;124:143-148.

9. Verdonck-de Leeuw IM, Hilgers F, Keus RB, et al. Multidimensional assessment of voice characteristics after ra- diotherapy for early glottic cancer. Laryngoscope 1999; 109:241-248.

10. Steiner W. Endoskopische Laserchirurgie der oberen Luft- und Speisewege. In: Steiner W, editor. Lasermikrochirurgie des Larynxkarzinoms. Stuttgart: Thieme; 1997. p 49-73.

11. Howell-Burke D, Peters LJ, Goepfert H, Oswald MJ. T2 glottic cancer: Recurrence, salvage and survival after devinitive radiotherapy. Arch Otolaryngol Head Neck Surg 1990;116:830-835.

12. Fein DA, Mendenhall WM, Parsons JT, Million RR. T1-T2 squamous cell carcinoma of the glottic larynx treated with radiotherapy: a multivariante analysis of variables potentially influencing local control. Int $\mathrm{J}$ Radiat Oncol Biol Phys 1993;25:605-611.

13. Stoeckli SJ, Guidiceli M, Schneider A, Huber A, Schmid S. Quality of life after treatment for early laryngeal carcinoma. Eur Arch Otorhinolaryngol 2001;258:96-99.

14. Peretti G, Nicolai P, Redaelli de Zillis LO, et al. Endoscopic $\mathrm{CO}_{2}$ laser excision for Tis, T1 and T2 glottic carcinomas: cure rate and prognostic factors. Otolaryngol Head Neck Surg 2000;123:124-131.

15. Ton-Van J, Lefebvre JL, Stem JC, Buisset E, CocheDequeant B, Vankemmel B. Comparison of surgery and radiotherapy in T1 and T2 glottic carcinomas. Am J Surg 1991;162:337-340.

16. Stoeckli SJ, Pawlik AB, Lipp M, Huber A, Schmid S. Salvage surgery after failure of nonsurgical therapy for carcinoma of the larynx and hypopharynx. Arch Otolaryngol Head Neck Surg 2000;126:1473-1477.

17. Moose BD, Greven KM. Definitive radiation management for carcinoma of the glottic larynx. In: McGuirt WF, editor. The Otolaryngologic clinics of North America. Philadelphia: Saunders; 1997. p 131-143.

18. Terhaard CH, Snippe K, Ravasz LA, van der Tweel I, Hordijk GJ. Radiotherapy in T1 laryngeal cancer: prognostic factors for locoregional control and survival, uniand multivariante analysis. Int J Radiat Oncol Biol Phys 1991;21:1179-1186. 